\title{
O papel da cultura organizacional e da aprendizagem para o sucesso da gestão do conhecimento
}

\section{Fernandez Kenji Inazawa}

\author{
Bibliotecário. Especialista em gestão do \\ conhecimento; Centro de Estudos Ambientais do \\ Cerrado da Reserva Ecológica do IBGE
}

Apesar das empresas buscarem ampliar seu conhecimento organizacional para obter vantagem competitiva, a Gestão do Conhecimento tem encontrado resistências, pois não há como deixar de trabalhar a cultura das organizações e seus processos de aprendizagem se há interesse real em se gerir o conhecimento. Dependendo do tipo de cultura predominante e da abertura que se dá ao aprender organizacional, uma empresa pode abri-se ou fechar-se ao processo de Gestão do Conhecimento. Este artigo, portanto, levanta a necessidade de se dar atenção à cultura organizacional e a seus processos de aprendizagem, para que a Gestão do Conhecimento seja bem sucedida. Cultura organizacional é um tema bastante amplo e complexo, que sofre influência tanto da cultura dos indivíduos que trabalham nas organizações, quanto da cultura do país em que as organizações se encontram. Sem querer esgotar o tema, buscou-se dar ênfase à questão da cultura organizacional em si e à sua relação com o sucesso da Gestão do Conhecimento. A aprendizagem organizacional foi apresentada como alternativa para flexibilizar a cultura e facilitar os processos de Gestão do Conhecimento.

Palavras-chave: Gestão do Conhecimento; Cultura Organizacional; Mudança Organizacional; Aprendizagem Organizacional. 


\title{
Understanding organizational culture and learning processes for the success of the knowledge management
}

\begin{abstract}
Although companies have been trying to widen their organizational knowledge in order to obtain competitive advantages, knowledge management finds opposition, for it is impossible not to work the culture of organizations and their learning processes if there is a real interest in managing knowledge. Depending on the type of the predominant culture and the opening that is given to organizational learning, a company can open or close itself to the process of learning management. So, this article raises the need of giving attention to the organizational culture and to its processes of learning so that knowledge management can be successful. Organizational culture is a very complex and extensive issue, which is influenced not only by the culture of the individuals who work in the organizations, but also by the culture of the country where organizations are settled. This article has also attempted to emphasize organizational culture itself and its relation to the success of Knowledge management. The organizational learning was presented as an alternative for facilitating the culture and the processes of knowledge management.
\end{abstract}

Keywords: Knowledge Management; Organizational Culture; Organizational Change; Organizational Learning.

Recebido em 19.08.2008 Aceito em 04.12.2009

\section{Breve exposição da relação entre trabalho e conhecimento no decorrer da história}

Fialho et al. (2006), reportando-se às origens da relação entre trabalho e conhecimento, começaram seu discurso dizendo que houve uma época na história da humanidade em que o conhecimento nas organizações se desenvolvia muito lentamente, período este compreendido entre a Pré-história e o século XIX. Nesta época, a base dos meios de produção era a manipulação de recursos naturais e a utilização da força física. Contudo, a partir da metade do século XIX, o grande progresso tecnológico e as invenções científicas já começam a acelerar o processo de geração de conhecimentos pelo uso mais acentuado da informação nos processos operacionais. 
Terra (1999, p. 10) apud Buoro et al. (2007, p.1) relata que "no final do século XIX, países ricos em recursos naturais, como a Argentina e o Chile, eram ricos, enquanto países sem muitos recursos naturais, como o Japão, estavam destinados a ser pobres". Com base no desenvolvimento científico e tecnológico, o Japão, apesar de todas as suas limitações em recursos naturais e intempéries, tais como abalos sísmicos freqüentes, através do conhecimento que desenvolveu, agregou valor a seus serviços e produtos superando o que constituía barreira ao seu desenvolvimento econômico. Singapura também soube aproveitar a nova onda econômica de progresso baseado no conhecimento, aproveitando os novos ciclos econômicos propiciados pela fabricação de produtos eletro-eletrônicos, de comunicações e de informática.

Com o aprimoramento cada vez maior da capacidade produtiva humana, dos meios de comunicação, das técnicas de produção e transmissão do conhecimento e da ciência, elevando o conhecimento ao nível de principal fator de produção do século XXI, surge a economia do conhecimento.

Teóricos como Toffler (1992), Kuhn (1996) e Lévy (1993), com o intuito de mostrar os vários estágios do progresso da civilização, tentaram sistematizar suas fases de evolução, classificando-as de acordo com diferentes aspectos, ou pontos de vista.

Toffler (1992), por exemplo, buscou compreender a evolução humana pelo prisma da tecnologia, dividindo-a em três ondas de transformação de sua capacidade produtiva: a primeira onda seria a revolução agrícola; a segunda, a industrial; e a terceira seria caracterizada pelos avanços da indústria eletrônica e da informática, das biociências, das conquistas espaciais e submarinas.

Kuhn (1996) analisa o progresso humano pelo viés da evolução da ciência. Relata que a revolução na ciência ocorreu por volta do século XVII, dando origem a um novo paradigma, o qual demorou cerca de 150 anos para se consolidar. Este paradigma foi chamado de "Paradigma newtoniano-cartesiano, mecanicista ou da simplificação".

Lévy (1993) foca na questão das "tecnologias do conhecimento" e divide a história do conhecimento em três momentos: oralidade primária, no qual o conhecimento era repassado de forma oral; escrita, que possibilitou o registro do conhecimento; e, por fim, o momento mediáticoinformático, que possibilitou construir um tipo de saber baseado em simulação, em previsões e em modelos.

Segundo Stewart (1998, p. 30), organizações pensadas com a intenção exclusiva de se obter capital financeiro ficaram no passado, pois as empresas já perceberam que o que gera os recursos necessários para se alcançar riquezas não são mais a terra e o trabalho físico, ou mesmo ferramentas mecânicas e fábricas, e sim ativos baseados em conhecimento. Apesar disto, Nonaka (2000, p. 28) assevera que as empresas ocidentais ainda não se abriram totalmente a este novo paradigma e, por isto, não modificaram a antiga visão de empresas como verdadeiras máquinas, que têm como objetivo a obtenção única e exclusiva de lucros. 
Fialho et al. (2006), no entanto, parecem discordar de Nonaka (2000), ao exporem que o trabalho tem mudado com muita rapidez, exigindo-se maior capacidade de cognição por parte dos trabalhadores, maior nível de aprendizagem e gestão efetiva do conhecimento gerado.

Portanto, hoje se vive a era da informação e do conhecimento, na qual cabe uma nova tarefa ao homem, ou seja, a de ser criativo e ter idéias; e o trabalho físico é realizado através de máquinas (SILVA; NEVES, 2003, p. 47).

O problema, agora, diz respeito a como gerir este conhecimento para que a empresa obtenha vantagem competitiva, porquanto o conhecimento organizacional depende do conhecimento individual, que precisa ser compartilhado entre os membros da organização. Em outras palavras, "o conhecimento organizacional por si só não existe, pois ele decorre da soma do conhecimento dos indivíduos que compõem a organização" (TEJERA DE RÉ et al., 2007, p. 2).

Costa e Gouvinhas (2005, p. 2) também comungam com a idéia de que a "[...] GC passa, essencialmente, pelo compartilhamento dos conhecimentos individuais para a formação do conhecimento organizacional". Ou seja, a pessoa que possui o conhecimento é quem decide se irá compartilhá-lo ou não, o que constitui um desafio a ser superado pela organização.

Mas, para que o conhecimento flua, e para que aconteça efetivamente

a Gestão do Conhecimento, é fundamental a criação de um ambiente propício, uma cultura de aprendizagem; o que se exigiria uma mudança de visão por parte dos líderes e dos liderados, que devem passar a enxergar a organização de uma forma diferente, ou seja, como estruturas de conhecimento e não somente de capital.

\section{A importância da cultura organizacional e da aprendizagem para o sucesso da gestão do conhecimento}

Para começar a discussão sobre a influência da cultura organizacional e da aprendizagem na Gestão do Conhecimento, é necessário entender, primeiramente, no que se constitui esta modalidade de gestão, situando-a e contextualizando-a. Após esta etapa, é preciso abordar os relacionamentos desta com a cultura organizacional, e também com a aprendizagem.

Apesar das empresas buscarem ampliar seu conhecimento organizacional para obter vantagem competitiva, a Gestão do Conhecimento tem encontrado resistências, pois não há como deixar de trabalhar a cultura das organizações e seus processos de aprendizagem se há interesse real em se gerir o conhecimento. Dependendo do tipo de cultura predominante e da abertura que se dá ao aprender organizacional, uma empresa pode abri-se ou fechar-se ao processo de Gestão do Conhecimento. Este artigo, portanto, levanta a necessidade de se dar 
atenção à cultura organizacional e a seus processos de aprendizagem, para que a Gestão do Conhecimento seja bem sucedida.

Cultura organizacional é um tema bastante amplo e complexo, que sofre influência tanto da cultura dos indivíduos que trabalham nas organizações, quanto da cultura do país em que as organizações se encontram. Sem querer esgotar o tema, buscou-se dar ênfase à questão da cultura organizacional em si e à sua relação com o sucesso da Gestão do Conhecimento. No que concerne à aprendizagem organizacional, esta foi apresentada como alternativa para flexibilizar a cultura da organização e facilitar os processos de Gestão do Conhecimento.

Moreira e Ferreira (2006, p. 1) conseguiram identificar na literatura sobre Gestão do Conhecimento (GC), em específico, nos trabalhos de (A) Sveiby (2001), (B) Barbosa e Paim (2003), (C) Davenport e Cronin (2000) e (D) Wilson (2002) aspectos teóricos que ajudam a entender melhor as várias formas de se ver o assunto:

(A) Em Sveiby (2001), por exemplo, a GC pode ser compreendida sob dois pontos de vista: foco nas novas tecnologias da informação e da comunicação, e foco nas pessoas.

(B) Para Barbosa e Paim (2003), quando o assunto é GC, basicamente, há três disciplinas principais: administração, ciência da computação e ciência da informação.

(C) Sob a ótica de Davenport e Cronin (2000), há, ainda, como relacionar GC a três domínios - KM1 (ciência da informação), KM2 (engenharia de processos) e KM3 (teoria organizacional).

(D) $\mathrm{E}$ as áreas que mais se dedicam ao estudo da Gestão do Conhecimento, para Wilson (2002), são a ciência da computação, a ciência da informação, a administração e a engenharia.

Segundo o modelo proposto por Terra (2001), a Gestão do Conhecimento pode ser entendida a partir de sete dimensões da prática gerencial, quais sejam: cultura organizacional, aprendizado com 0 ambiente, visão e estratégia da alta gerência, estruturas organizacionais, políticas de recursos humanos, sistemas de informação e comunicação, e mensuração de resultados. Para este artigo, destacam-se duas dimensões do modelo supracitado: a cultura organizacional e o aprendizado com o ambiente.

Há muitas maneiras de se conceituar Gestão do Conhecimento, mas uma definição bem interessante, exposta por Tejera de Ré et al. (2007, p. 2 ), considerando-a do ponto de vista de sua abrangência e por manter relação com a aprendizagem organizacional, é que a Gestão do Conhecimento visa a desenvolver sistemas e processos que englobem desde a criação, a identificação, a integração, a recuperação e o compartilhamento do conhecimento, até a sua utilização pela empresa, "com o objetivo de aumentar o aprendizado individual e grupal e, dessa forma, criar competências exclusivas que diferenciarão a organização no mercado em que compete".

Partindo-se desta conceituação de Gestão do Conhecimento, percebe-se sua complexidade, que vai muito além de uma simples solução de TI. O objetivo da GC é, antes, auxiliar a aprendizagem em âmbito 
individual e coletivo, e, portanto, deve ter o foco no "ser que aprende", ou seja, no homem, e não na tecnologia, pelo fato de ser ele "o único sujeito de interlocução entre os diferentes ambientes - interno e externo - e o agente de qualquer processo de mudança" (TEIXEIRA; PIZOLOTTO; LAMPERT, 2004, p. 3897).

Para Nonaka e Takeuchi (1997), o que gera o conhecimento organizacional é a interação entre conhecimentos tácitos e explícitos. No entanto, para que possa ser compartilhado há que se ter uma cultura de compartilhamento do conhecimento na organização, que incentive o indivíduo a participar deste processo de troca. Em geral, empresas que passaram por reengenharia ou downsizing, por exemplo, de acordo com Candido e Junqueira (2006, p. 13), podem vir a desenvolver culturas organizacionais que tendem a acirrar a competitividade entre os membros da equipe, levando-os a não quererem compartilhar abertamente o que sabem.

Algumas pesquisas sobre GC no Brasil estão levantando informações interessantes sobre o papel crucial da cultura organizacional, em especial o aspecto do compartilhamento do conhecimento dentro das organizações. Em 2004 e 2005, Moreira e Ferreira (2006, p. 8), com o objetivo geral de analisar as características da prestação de serviços de consultoria em GC, realizaram uma pesquisa com 19 organizações de consultoria (de um universo composto de 79 organizações) e chegaram à seguinte conclusão:

Em relação aos pontos fundamentais ao sucesso de um projeto de GC, o alinhamento da GC à estratégia organizacional foi o mais assinalado (aproximadamente $79 \%$ das organizações), seguido pela existência ou desenvolvimento de cultura de compartilhamento de informações e conhecimentos (aproximadamente 68\% das organizações) e pelo apoio da alta administração ao projeto (aproximadamente $63 \%$ das organizações); apenas dois pontos não foram indicados: a gestão dos recursos humanos e a justificação da implantação da GC a todos os membros da organização (MOREIRA; FERREIRA, 2006, p. 8).

Nota-se, portanto, o peso que se deve dar ao fator "cultura organizacional" no processo de implementação, com sucesso, da Gestão do Conhecimento. Este processo poderá vir a se tornar bastante difícil se a cultura organizacional não privilegiar o desenvolvimento de uma cultura voltada à aprendizagem e ao compartilhamento do conhecimento.

Mas há que se pensar, amiúde, no incentivo ao aprendizado pessoal e organizacional, que valorizará o conhecimento dentro da organização e não somente o compartilhamento. Em outras palavras, deve haver um tipo de cultura que seja mais propícia ao aprendizado, e, por conseguinte, à Gestão do Conhecimento; ou seja, "se as pessoas começam a compartilhar idéias a respeito de assuntos que consideram importantes, esse mesmo compartilhamento de idéias cria uma cultura de aprendizado, transformando o conhecimento no principal ativo da organização" (COSTA; GOUVINHAS, 2005, p. 2). 
A cultura da organização é tão importante, que Costa e Gouvinhas (2005) a citam como um dos três alicerces que devem preparar uma empresa para as iniciativas e estratégias de Gestão do Conhecimento. Os outros dois alicerces são: o mapeamento dos processos, "pois uma empresa precisa conhecer como ela mesma funciona, ou seja, como são realizadas suas operações, seus negócios e suas atividades" (p. 2); e a Tecnologia da Informação, que, pelo barateamento dos custos atuais, não mais constitui uma dificuldade para implantar a GC.

Há uma grande diferença entre valorizar o conhecimento em si e valorizar a pessoa que o detém, considerando-se as barreiras culturais que destes posicionamentos podem surgir ao compartilhamento e à Gestão do Conhecimento. No primeiro caso, o conhecimento flui dentro da organização de forma a criar mais conhecimento; no segundo, a idéia básica é a de que a retenção do conhecimento simboliza a garantia de emprego.

Os funcionários podem, por exemplo, sentir que o seu conhecimento é crucial para valorizá-lo como empregado e, portanto, para sua permanência na organização. Em tal circunstância é comum que os usuários relutem em compartilhar seu conhecimento (CANDIDO; JUNQUEIRA, 2006, p.13).

Tendo-se em vista que as organizações contemporâneas precisam inovar cada vez mais, é de grande relevância compreender esta vertente valiosa para o sucesso do processo de inovação, a cultura organizacional. A despeito de toda a informação que se tenha sobre a necessidade atual de se gerir o conhecimento da organização, querer fazer Gestão do Conhecimento sem entender, em profundidade, a cultura da organização pode levar ao fracasso desta empreitada e, até mesmo, aumentar a resistência ao novo, acirrando ainda mais rotinas defensivas. É por isto que Costa e Gouvinhas (2005, p. 4) enfatizam a mudança cultural como essencial para a implementação da GC, e salientam que "nenhuma transformação ocorre sem sofrer resistências. As pessoas têm uma tendência natural a reagir à mudança". 


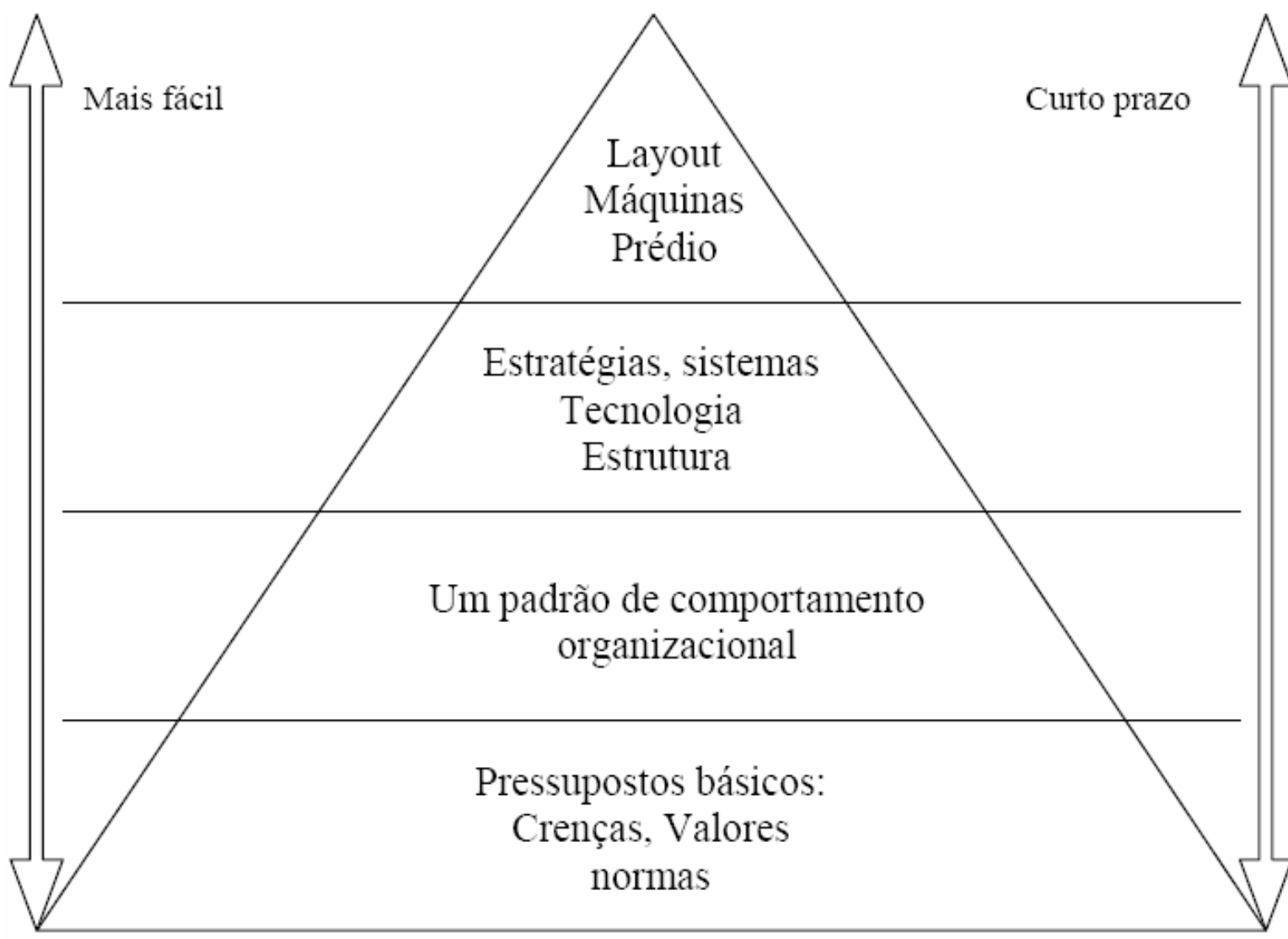

Mais difícil

Longo prazo

FIGURA 1 - Pirâmide da mudança cultural

Fonte: Adaptado de MONTEIRO; VENTURA; CRUZ (1999) apud ALVES (2006).

É essencial compreender como se processam as mudanças. Através da FIG. 1, pode-se ter uma idéia de como se dá a dinâmica de mudança nas organizações. inferior.

A pirâmide está dividida em quatro níveis: superior, intermediário e

O nível superior da pirâmide é composto de um degrau apenas, que, por analogia, seria como a ponta de um iceberg, e se refere à estrutura material da organização, composta, por exemplo, pelo prédio em que se encontra a empresa e seu layout, suas máquinas, mobiliário e toda a sorte de bens móveis e imóveis. A ponta do iceberg é a parte mais visível da empresa, e também é a porção que, em curto prazo, é mais fácil de ser alterada.

O nível intermediário é composto por dois degraus. De abaixo para cima, tem-se o primeiro degrau representado pela parte lógica da organização. São as tecnologias, os sistemas, as estratégias, as políticas, as normas, entre outras estruturas em que os negócios, bem como as pessoas que trabalham nas empresas, se apóiam para executar suas funções. Apesar de este nível estar em direção à base da pirâmide, que é onde há o maior nível de resistência às mudanças, também comporta certa fluidez para mudanças e adaptações, como o primeiro nível. 
No segundo degrau do nível intermediário, começa a ficar mais evidente a dificuldade de se trabalhar com mudanças. Neste ponto, já se tem um padrão de comportamento organizacional estabelecido. Trabalhar comportamento é bem mais difícil e leva muito mais tempo do que os aspectos concernentes ao primeiro degrau, nível superior, e segundo degrau, primeiro nível intermediário da pirâmide.

Chega-se, finalmente, à base da pirâmide. Esta é formada pelas crenças, valores e normas que formam a cultura organizacional. É a parte mais difícil de ser mudada na organização e é a que move toda a pirâmide. Para onde a base se mover, a pirâmide toda terá que acompanhar.

Sob o enfoque das resistências, para Salvador (2002) apud Costa e Gorvinhas (2005, p. 5) há três tipos de perfis de pessoas na organização, que, quando identificados, são úteis ao desenvolvimento de estratégias de comunicação e envolvimento, facilitando, deste modo, a quebra das barreiras culturais:

- Os resistentes. Resistem a qualquer tipo de mudança, que dirá a um novo sistema. Podem ser resistentes, por falta de habilidades com a tecnologia, insegurança quanto ao futuro de seu emprego ou simplesmente são os populares "do contra". Mantenha esse grupo informado, treinado e se possível não o deixe "contaminar" o projeto. 0 grande problema é quando esses resistentes estão na alta cúpula da administração, o que provavelmente tornará a implantação da GC mais difícil. Mas esse grupo tem um papel importante, pois ao ter de defender e justificar seus planos eles se tornarão mais consistentes.

- Os neutros. A grande maioria da população das organizações. Esse grupo deve ser conquistado com informações precisas ao longo de todo o projeto de implementação da GC, como relatos de casos de sucesso de outras organizações ou mesmo com projetos pilotos realizados na empresa e principalmente com a demonstração clara de como o novo sistema vai melhorar a vida deles no trabalho e a produtividade na empresa.

- Os inovadores. Esses provavelmente estarão na equipe que implantará a GC ou serão os grandes patrocinadores de qualquer iniciativa nova na empresa. Tenha-os como os grandes multiplicadores do projeto.

Costa e Gouvinhas (2005, p. 2) consideram que "a mudança cultural é um pré-requisito para a implementação da GC". Uma mudança de cultura, entretanto, não deve ser vista como "criação de uma nova cultura". Para Schein (2000), por exemplo, não há como criar uma nova cultura, mas estudá-la em sua profundidade, ampliando a compreensão de seus valores para, depois, propor outros valores e novas idéias. Este procedimento servirá como guia para a "evolução cultural", que poderá ocorrer caso se aceite e adote os novos comportamentos advindos dos valores provenientes do processo da transformação organizacional, que, ainda segundo Costa e Gouvinhas (2005, p. 4), depende do tipo de cultura organizacional. Este processo "pode ser longo e tortuoso. Pois a abertura cultural não está inerente à maioria das pessoas". 
As barreiras culturais à GC também podem advir da ausência de visão compartilhada, do fato de esse tipo de gestão não despertar interesse ou fazer sentido para as pessoas, ou seja, da inexistência de envolvimento verdadeiro, gerando falta de conexão entre os interesses pessoais e os objetivos propostos. Através da Aprendizagem Organizacional, Senge (2000) mostra como é possível compartilhar visões, objetivos e conhecimento no ambiente da empresa, ao focar desejos, crenças e valores individuais. Compartilhar visões significa "reunir pessoas em torno de uma identidade e senso de destino comuns".

Se não houver uma cultura organizacional que estimule as pessoas a desenvolverem relacionamentos e a socializarem seus conhecimentos, a probabilidade de guardarem para si seus conhecimentos será maior. $\mathrm{E} o$ velho paradigma privilegia o jeito formal de se trocar conhecimento, que é realizando reuniões de trabalho, reduzindo a conexão entre as pessoas e a exteriorização do conhecimento. Existem, ainda, empresas que chegam a reprimir o contato entre os trabalhadores, impondo regras rígidas de controle; não permitem, por exemplo, que os funcionários troquem idéias nos corredores, com medo de ocorrer algum motim, pois sabem que suas políticas de recursos humanos não são boas o suficiente para a criação de vínculos afetivos com seus empregados. Portanto, mudanças culturais dentro das organizações urgem.

Meios informais são boas fontes para se criar conexão entre os membros de uma empresa, permitindo que estes troquem idéias e conversem nos corredores, no intervalo para o café, etc. Mas algumas empresas entendem que estas práticas são um desperdício de tempo, por pensarem que a troca de idéias somente pode ocorrer em reuniões formais. Ponderam Davenport e Prusak (1998) apud Tejera de Ré et al. (2007, p. 5).

Atualmente, a necessidade de se aprender com rapidez é uma habilidade primordial, mas para que esse processo de aprendizagem acelerada aconteça, mudanças nos pressupostos básicos compartilhados pelas pessoas precisam ocorrer. Os líderes desempenham um papel essencial, ao estimularem uma cultura de aprendizagem, recompensando aqueles comportamentos desejados (ARGYRIS, 2001, p. 7 apud TEJERA DE RÉ et al., 2007, p. 6).

Até agora, o foco principal do presente artigo foi a chamada microcultura, ou cultura organizacional. Esta é influenciada pela cultura trazida pelas pessoas que trabalham na empresa, bem como pela cultura do país, que é a macrocultura nacional. Também entram vários outros fatores culturais e econômicos de influência para a microcultura, como o mercado no qual a empresa atua, o tipo de liderança, etc., desenvolvimento de culturas organizacionais voltadas à inovação, à experimentação, ao aprendizado contínuo e à obtenção de resultados no longo prazo (e não apenas no curto prazo)

Hofstede (1997) apud Tejera de Ré et al. (2007, p. 5) realizou uma pesquisa sobre a influência da macrocultura nacional sobre a microcultura 
organizacional, em uma multinacional que atuava em diversos países, e constatou "diferenças significativas entre os profissionais de diferentes países provocadas pela influência da cultura nacional sobre a cultura organizacional".

A mesma empresa, com filiais em outros estados ou países, também pode ter estilos diferentes de gerenciamento e cultura organizacional, dependendo de sua estratégia empresarial.

Como exemplo, Bisson (2002, p. 18) diz que, para um funcionário que participou de sua pesquisa de GC na Oracle do Brasil, as iniciativas de GC obtiveram mais sucesso na Europa do que no Brasil, principalmente pelas diferenças culturais, e aponta estas diferenças: "a Oracle do Brasil tem uma cultura fortemente influenciada pela cultura de vendas, o que não é o caso das representações da Oracle na Europa".

Bisson (2002, p. 18) pondera e complementa que "a cultura de vendas da Oracle do Brasil facilita e dificulta, ao mesmo tempo, iniciativas de Gestão do Conhecimento. Ela traz uma grande criatividade e flexibilidade e, também, dificulta a aprendizagem e o compartilhamento". No caso da Oracle, a GC sofreu impacto diferente de acordo com as culturas organizacionais locais, oriundas de estratégias empresariais diferentes. No caso, como filial, a estratégia da Oracle do Brasil está centrada em vendas.

Alguns autores dizem que, apesar de o ambiente organizacional brasileiro estar passando por mudanças significativas, ainda há traços culturais que dificultam a implementação de técnicas de gestão mais modernas, como a gestão do conhecimento. Citam-se, como exemplos de vivências organizacionais que dificultariam a GC, o personalismo e a concentração de poder. Conjugados, eles reforçam o paternalismo e as estruturas organizacionais verticalizadas, que, por conseguinte, acabam gerando a centralização das decisões e uma postura de espectador nos trabalhadores. Muitos têm medo de errar e perder o emprego, e se limitam a executar o que a sua função exige, restringindo a aprendizagem e diminuindo o conhecimento.

Um sistema de GC requer flexibilidade organizacional, ou seja, estruturas organizacionais horizontalizadas, compartilhamento de poder e um clima de confiança e segurança que incentive a inovação e a experimentação pelo método de tentativa e erro, além do desenvolvimento da capacidade de aprendizagem das pessoas que trabalham nas empresas.

Em suma, a importância da cultura organizacional se refere, basicamente, à promoção de uma maior abertura, para que as pessoas troquem conhecimento entre si de forma mais ampla, assim permitindo que as elas inovem, tenham espaço para a experimentação, o aprendizado contínuo e a obtenção de resultados a longo prazo. E o papel da aprendizagem é aumentar o conhecimento dentro da organização através da criatividade, da experimentação e da inovação, elementos tão essenciais para a solução de problemas e a criação de novos produtos e serviços. 
Há outras questões que poderiam ser estudadas com maior profundidade e que não fazem parte do objetivo definido neste artigo, tais como a formação dos diferentes povos de cada país e suas peculiaridades culturais, ou seja, as crenças, valores, hábitos e tradições que influem na cultura organizacional, entre outras.

\section{Conclusão}

Pela literatura pesquisada, há muito que se fazer em termos de Gestão do Conhecimento, e muito que se mudar culturalmente dentro das empresas. É necessário estudar para entender melhor, não só as relações das pessoas com a tecnologia nos ambientes coorporativos, mas suas relações entre si, seus comportamentos, motivações, etc.

Observou-se, também, um elo importante entre a cultura das organizações e questões que influenciariam o sucesso da Gestão do Conhecimento. É necessário trabalhar para desenvolver um tipo de cultura favorável à GC, o que geraria maior nível de aprendizado e de conformidade com as rápidas mudanças de um mundo em acelerada transformação. Uma cultura organizacional voltada para a aprendizagem pode facilitar o processo de GC na empresa.

Percebeu-se que desenvolver esta cultura de GC vai muito além de abrir-se para um novo tipo de gestão; diz respeito à transformação da organização como um todo. Ou, como afirma Candido e Junqueira (2006, p. 22):

Significa não só atuar na superfície da organização, mas reavaliar seus pressupostos e crenças, tidas como verdade, para isto as organizações que se pretendem gestoras do conhecimento precisam, efetivamente, apoiar-se em um quadro referencial que inclua tecnologia e pessoas, técnica e comportamento humano.

\section{Referências}

ALVES, M. L. F. P. M. Gestão do conhecimento e seus impactos culturais no SERPRO: a percepção de gerentes e empregados da Regional Recife. 2006. 152f. Dissertação (Mestrado Profissional em Gestão Pública para o Desenvolvimento do Nordeste) - UFPE, Recife, 2006.

ARGYRIS, C. Sobre el aprendizaje organizacional. México (D.F.): Oxford, 2001 apud TEJERA DE RÉ, C. A. et al. O impacto da cultura organizacional no processo de Gestão do Conhecimento. In: ENCONTRO NACIONAL DE ENGENHARIA DE PRODUÇÃO, 27., Foz do Iguaçu, PR, Brasil, 09 a 11 de outubro de 2007. Anais... Foz do Iguaçu, 2007.

BARBOSA, R. R.; PAIM, Í. Da gerência de recursos informacionais à gestão do conhecimento. In: PAIM, Í. (Org.). A gestão da informação e do conhecimento. Belo Horizonte: Escola de Ciência da Informação da UFMG, 2003. cap. 1, p. 07-31 apud MOREIRA, D. A.; FERREIRA, M. A. T. Teoria e prática em gestão do conhecimento (GC): um olhar exploratório sobre as 
empresas de consultoria em GC atuantes no Brasil. GT 3: Mediação, Circulação e Uso da informação. In: ENCONTRO NACIONAL EM PESQUISA EM CIÊNCIA DA INFORMAÇÃO (ENANCIB), 7., Marília, SP, 19 a 22 de Novembro de 2006. Anais... Marília: ANCIB, 2006. Disponível em: <http://www.portalppgci.marilia.unesp.br/enancib/viewpaper.php?id=266 $>$. Acesso em: 04 ago. 2008.

BISSON, A. Estudo de gestão do conhecimento na Oracle do Brasil. 2002. 28 f. Monografia (MBA em Tecnologia da Informação) - Centro Universitário Nove de Julho, São Paulo, 2002. Disponível em: <http://www.adrich.net/KM.pdf>. Acesso em: 11 ago. 2008.

BUORO, G. Compartilhamento de conhecimento: um estudo sobre os fatores facilitadores. In: ENCONTRO DA ANPAD, 31., Rio de Janeiro, RJ, 22 a 27 de setembro de 2007. Anais... Rio de Janeiro, ANPAD, 2007. Disponível

em: <http://www.ecocidades.org/repositorio/File/artigo_04.pdf>. Acesso em: 31 jul. 2008.

CANDIDO, T. E.; JUNQUEIRA, L. A. P. Influência da Cultura Organizacional na Gestão e Compartilhamento do Conhecimento. eGesta, v. 2, n. 1, p. 524, jan.-mar./2006. Disponível em: < http://www.unisantos.br/mestrado/gestao/egesta//artigos/52.pdf>.

Acesso em: 30 jul. 2008.

COSTA, P. E. C.; GOUVINHAS, R. P. Gestão do Conhecimento: quebrando o paradigma cultural para motivar o compartilhamento do conhecimento dentro das organizações. 2005. Disponível em: <http://www.biblioteca.sebrae.com.br/bds/BDS.nsf/A73A5081143934CC0 3256FA600134AE5/\$File/Gestao\%20do\%20Conhecmiento\%20e\%20Cultur a\%200rganizacional.pdf>. Acesso em: 05 ago. 2008.

DAVENPORT, E.; CRONIN, B. Knowledge management: semantic drift or conceptual shift? [On-line]. 2000. Disponível em: <http://www.alise.org/conferences/conf00_Davenport-

Cronin_paper.htm>. Acesso em: 19 jul. 2004 apud MOREIRA, D. A.; FERREIRA, M. A. T. Teoria e prática em gestão do conhecimento (GC): um olhar exploratório sobre as empresas de consultoria em GC atuantes no Brasil. GT 3: Mediação, Circulação e Uso da informação. In: ENCONTRO NACIONAL EM PESQUISA EM CIÊNCIA DA INFORMAÇÃO (ENANCIB), $7 .$, Marília, SP, 19 a 22 de Novembro de 2006. Anais... Marília: ANCIB, 2006. Disponível em: <http:// www.portalppgci.marilia.unesp.br/enancib/viewpaper.php?id=266>. Acesso em: 04 ago. 2008.

FIALHO, F. A. et al. Gestão do conhecimento e aprendizagem: As estratégias competitivas da sociedade pós-industrial. Florianópolis: Visual Books, 2006. v. 01. 196 p.

KUHN, T. S. A estrutura das revoluções científicas. $4^{a}$ ed. São Paulo: Perspectiva, 1996. 
LÉVY, P. As tecnologias da inteligência: o futuro do pensamento na era da informática. Rio de Janeiro: Editora 34, 1993.

MONTEIRO, C. D. B.; VENTURA, E. C.; CRUZ, P. N. Cultura e mudança organizacional: em busca da compreensão sobre o dilema das organizações. Caderno de Pesquisas em Administração, São Paulo, v. 1, n. 8, p. 69-80, 1999 apud ALVES, M. L. F. P. M. Gestão do conhecimento e seus impactos culturais no SERPRO: a percepção de gerentes e empregados da Regional Recife. 2006. 152f. Dissertação (Mestrado Profissional em Gestão Pública para o Desenvolvimento do Nordeste) UFPE, Recife, 2006.

MOREIRA, D. A.; FERREIRA, M. A. T. Teoria e prática em gestão do conhecimento (GC): um olhar exploratório sobre as empresas de consultoria em GC atuantes no Brasil. GT 3: Mediação, Circulação e Uso da informação. In: ENCONTRO NACIONAL EM PESQUISA EM CIÊNCIA DA INFORMAÇÃ̃O (ENANCIB), 7., Marília, SP, 19 a 22 de Novembro de 2006. Anais... Marília: ANCIB, 2006. Disponível em: <http:// www. portalppgci. marilia.unesp.br/enancib/viewpaper. php?id=266>.

Acesso em: 04 ago. 2008.

NONAKA, I. A empresa criadora de conhecimento. In: HARVARD BUSSINESS REVIEW. Gestão do Conhecimento. Rio de Janeiro: Campus, 2000. p. 27-49.

NONAKA, I.; TAKEUCHI, H. Criação de conhecimento na empresa. Rio de Janeiro: Campus; 1997.

SALVADOR, A. S. Como implementar um projeto de tecnologia da informação para RH. In: BOOG, G.; BOOG, M. (Coords.). Manual de gestão de pessoas e equipes. São Paulo: Gente, 2002. v. 1, cap. 14 apud COSTA, P. E. C.; GOUVINHAS, R. P. Gestão do Conhecimento: quebrando o paradigma cultural para motivar o compartilhamento do conhecimento dentro das organizações. 2005. Disponível em: <http://www.biblioteca.sebrae.com.br/bds/BDS.nsf/A73A5081143934CC0 3256FA600134AE5/\$File/Gestao\%20do\%20Conhecmiento\%20e\%20Cultur a\%200rganizacional.pdf>. Acesso em: 05 ago. 2008.

SCHEIN, E. H. Organizational culture \& leadership. 2000. Disponível em: <http://www.tnellen.com/ted/tc/schein.html>. Acesso em 30 jul. 2008.

SENGE, P. A quinta disciplina: a dança das mudanças. Rio de Janeiro: Campus, 2000

SILVA, R. V.; NEVES, A. (Orgs). Gestão de empresas na era do conhecimento. Lisboa: Sílabo, 2003.

STEWART, T. A. Capital intelectual: a nova vantagem competitiva das empresas. 8. ed. Rio de Janeiro: Campus, 1998.

SVEIBY, K. What is knowledge management? 2001. Disponível em: <http://www.sveiby.com/articles/KnowledgeManagement.html>. Acesso em: 27 maio de 2004 apud MOREIRA, D. A.; FERREIRA, M. A. T. Teoria e 
prática em gestão do conhecimento (GC): um olhar exploratório sobre as empresas de consultoria em GC atuantes no Brasil. GT 3: Mediação, Circulação e Uso da informação. In: ENCONTRO NACIONAL EM PESQUISA EM CIÊNCIA DA INFORMAÇÃO (ENANCIB), 7., Marília, SP, 19 a 22 de Novembro de 2006. Anais... Marília: ANCIB, 2006. Disponível em: <http://www.portalppgci.marilia.unesp.br/enancib/viewpaper.php?id=266 $>$. Acesso em: 04 ago. 2008.

TEIXEIRA, E. B.; PIZOLOTTO, M. F.; LAMPERT, A. L. A gestão do conhecimento em uma organização bancária: competências profissionais num contexto de mudanças. In: ENCONTRO NACIONAL DE ENGENHARIA DE PRODUÇÃO, 24., Florianópolis, SC, Brasil, 03 a 05 de nov de 2004. Anais... Florianópolis, 2004.

TEJERA DE RÉ, C. A. et al. O impacto da cultura organizacional no processo de Gestão do Conhecimento. In: ENCONTRO NACIONAL DE ENGENHARIA DE PRODUÇÃO, 27., Foz do Iguaçu, PR, Brasil, 09 a 11 de outubro de 2007. Anais... Foz do Iguaçu, 2007.

TERRA, J.C.C. Gestão do conhecimento: aspectos conceituais e estudo exploratório sobre as práticas de empresas brasileiras.1999. Tese (Doutorado) - Escola Politécnica, USP, São Paulo, 1999 apud BUORO, G. Compartilhamento de conhecimento: um estudo sobre os fatores facilitadores. In: ENCONTRO DA ANPAD, 31., Rio de Janeiro, RJ, 22 a 27 de setembro de 2007. Anais... Rio de Janeiro, ANPAD, 2007. Disponível em: <http://www.ecocidades.org/repositorio/File/artigo_04.pdf>. Acesso em: 31 jul. 2008.

. Gestão do conhecimento: o grande desafio empresarial. 3 ed. São Paulo: Negócio, 2001.

TOFFLER, A. A terceira onda. 18a ed. Rio de Janeiro: Record, 1992.

WILSON, T. D. The nonsense of "knowledge management". Information Research, v. 8, n. 1, October 2002. Disponível em: <http://InformationR.net/ir/8-1/paper144.html>. Acesso em: 31 maio 2004 apud MOREIRA, D. A.; FERREIRA, M. A. T. Teoria e prática em gestão do conhecimento (GC): um olhar exploratório sobre as empresas de consultoria em GC atuantes no Brasil. GT 3: Mediação, Circulação e Uso da informação. In: ENCONTRO NACIONAL EM PESQUISA EM CIÊNCIA DA INFORMAÇÃO (ENANCIB), 7., Marília, SP, 19 a 22 de Novembro de 2006. Anais... Marília: ANCIB, 2006. Disponível em: <http:// www. portalppgci.marilia.unesp.br/enancib/viewpaper.php?id=266>.

Acesso em: 04 ago. 2008. 